\title{
Hybrid Perovskites with Larger Organic Cations Reveal Autocatalytic Degradation Kinetics and Increased Stability under Light
}

Christie L.C. Ellis, Hamza Javaid, Emily C. Smith, D. Venkataraman*

University of Massachusetts Amherst, Department of Chemistry, 690 N Pleasant St, Amherst, MA, 01003

Email: dv@chem.umass.edu

SUPPORTING INFORMATION 
a.

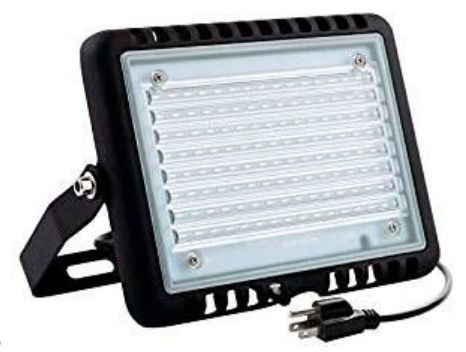

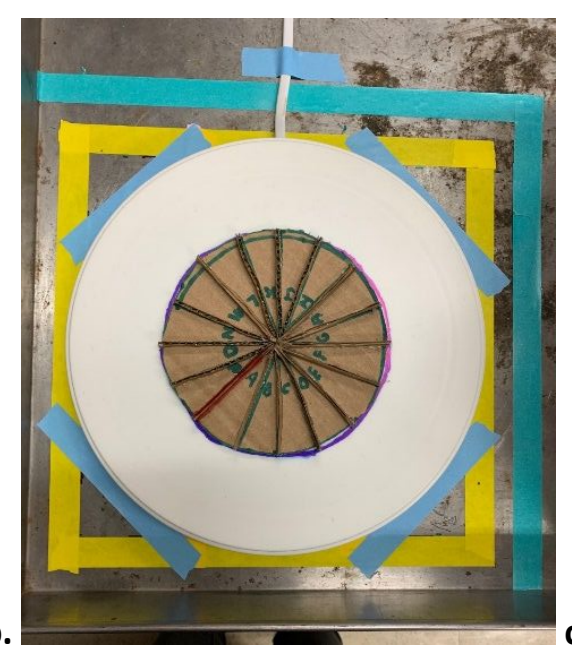

b.

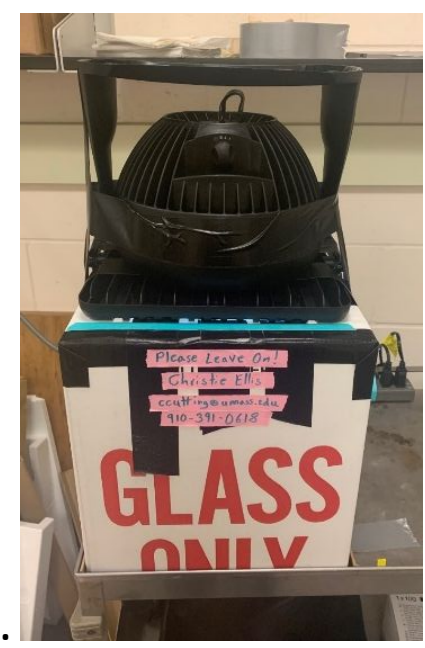

Figure S1: Set-up for light-induced degradation kinetics experiments. a. LED array used as light source for experiments. b. Turntable (white) with wedge to hold samples. Turntable rotated at 1.6 rpm to ensure even illumination of all samples. c. Samples on turntable placed beneath housing which blocked out other light with LED array shining down from top and cooling fan on top of LED. Temperature under light with cooling fan was $35^{\circ} \mathrm{C}$. 


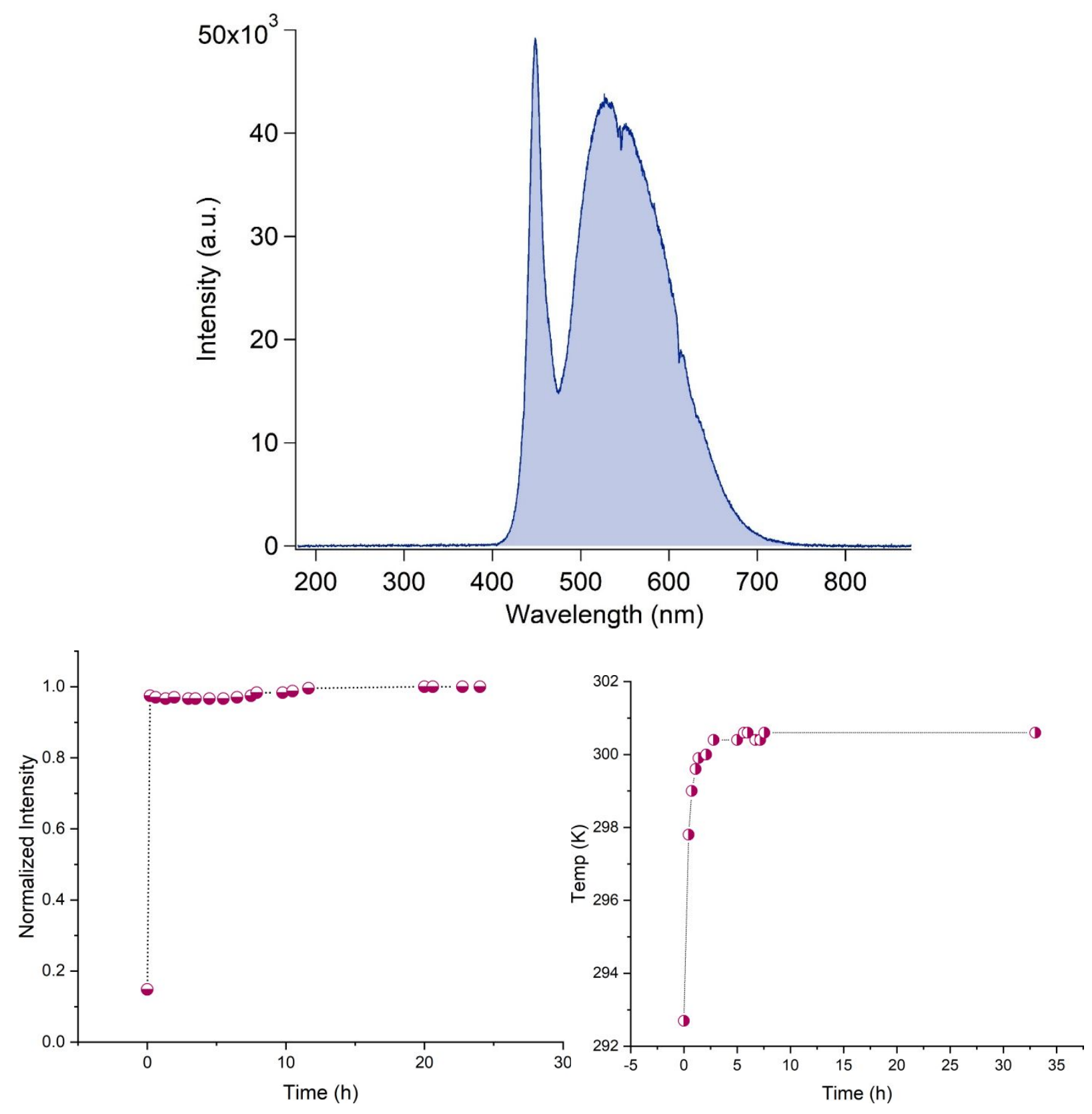

Figure S2: (top) Spectrum of LED lamp used in degradation experiments. Intensity was $\sim 5$ Sun. (left) intensity as a function of time and (right) temperature as a function of time. 


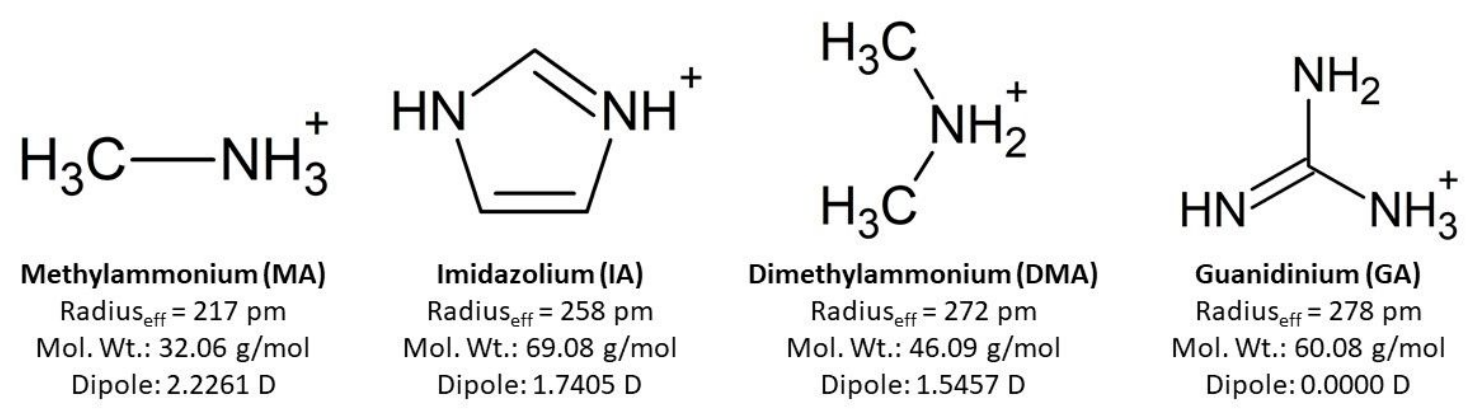

Figure S3: Properties of large organic ammonium cations used in this work. 

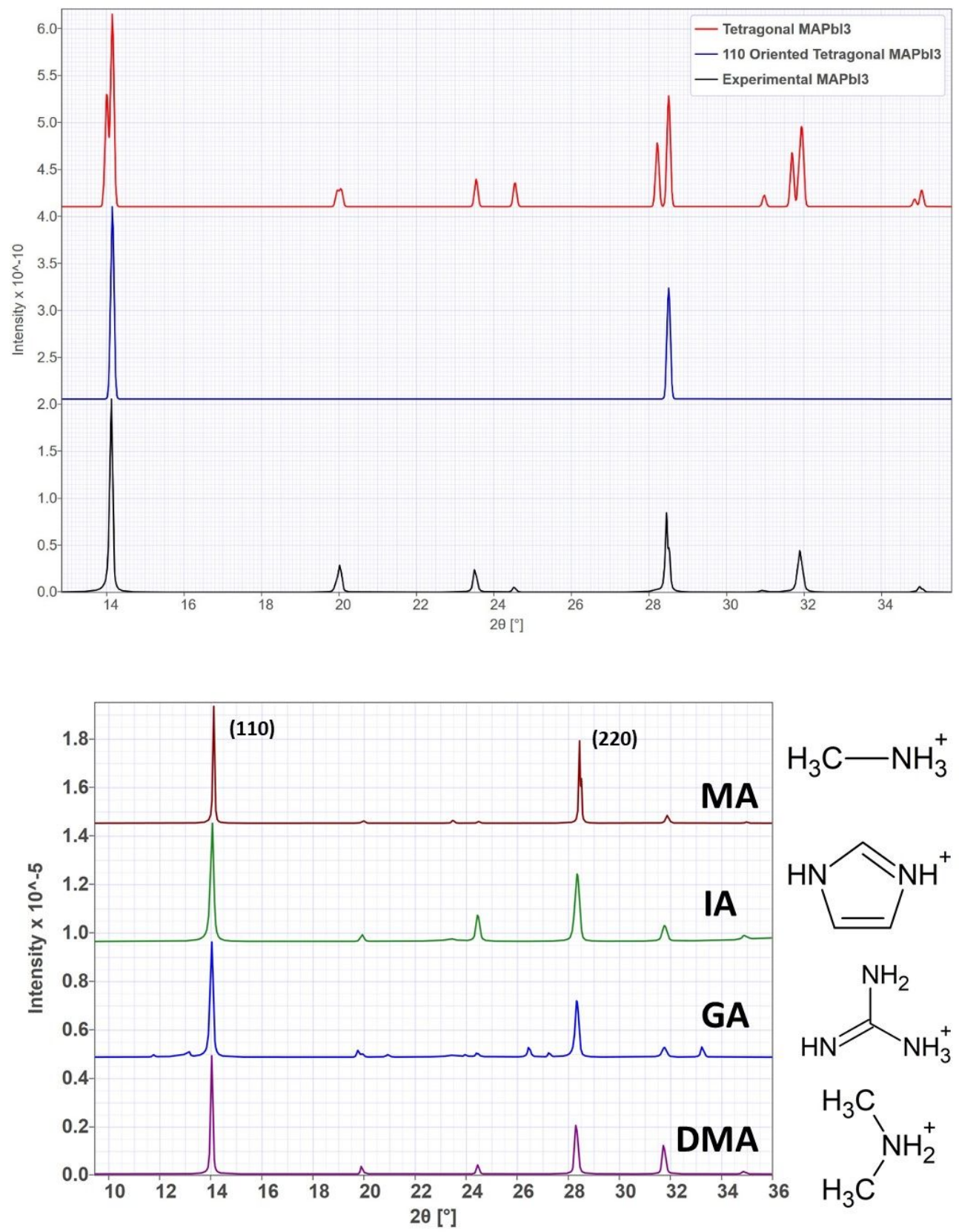

Figure S4: (top)PXRD patterns predicted for randomly oriented tetragonal $\mathrm{MAPbl}_{3}$ (top, red), 110oriented tetragonal $\mathrm{MAPbl}_{3}$ (middle, blue), and our experimental $\mathrm{MAPbl}_{3}$ film (bottom, black). Our samples show preferential (but not complete) orientation in the 110 direction. (bottom) PXRD patterns of $\mathrm{MAPbl}_{3}$ and $10 \%$ substitution of IA, GA, and DMA. All four maintained the $\mathrm{MAPbl}_{3}$-like tetragonal 3D perovskite structure. The films were oriented in the $<110>$ direction, so the $(110)$ and $(220)$ peaks were most prominent and present in all four samples. The (110) peak was used to quantify degradation of the 3D perovskite structure. Small $\mathrm{Pbl}_{2}$ impurity peaks arising from incomplete starting material conversion are visible in GA pattern. 

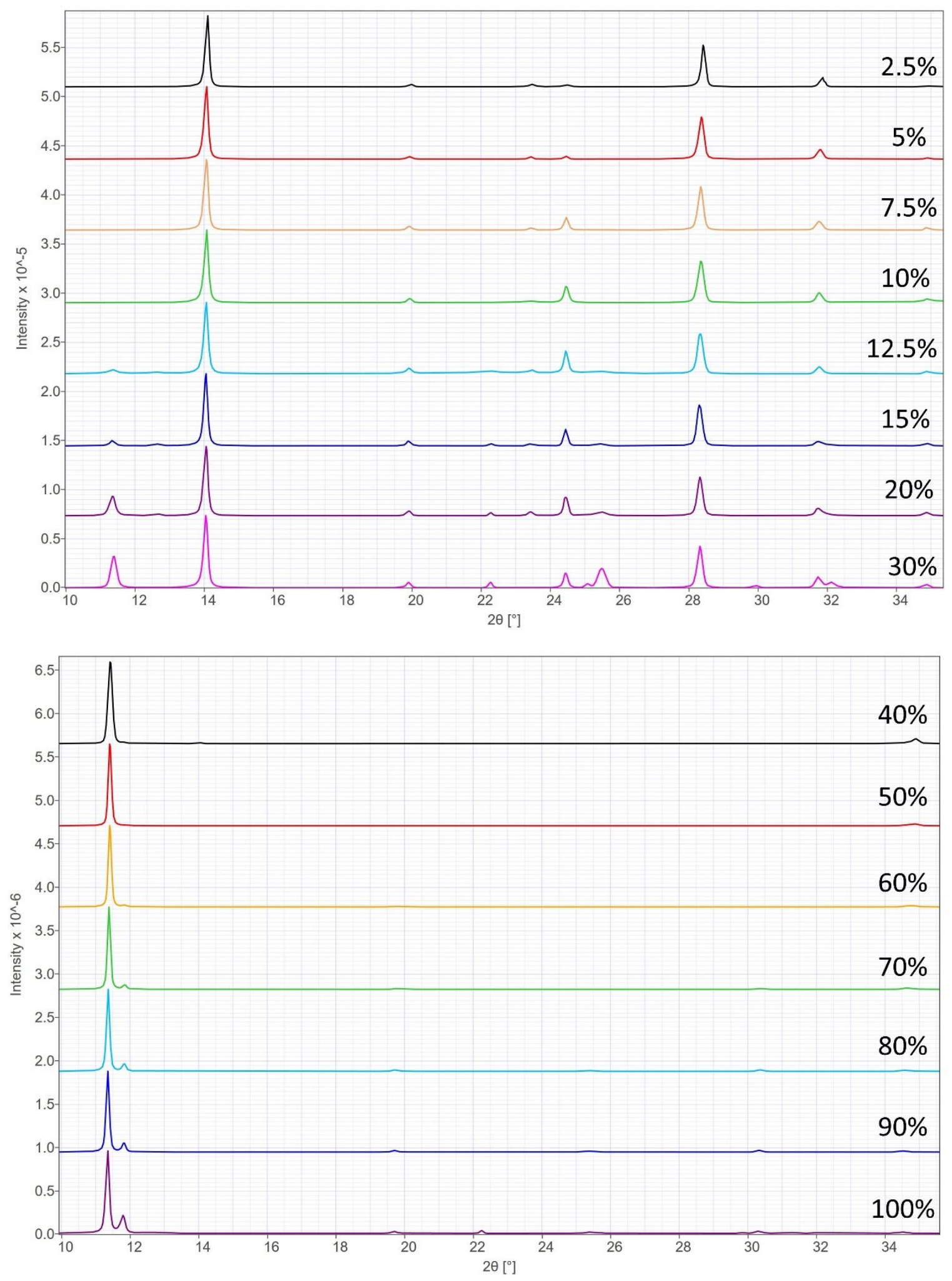

Figure S5: PXRD patterns of varying substitution percentages of IA (imidazolium) for MA (methylammonium) in $\mathrm{MA}_{1-x} \mid \mathrm{A}_{\mathrm{x}} \mathrm{Pbl}_{3}$ materials. 

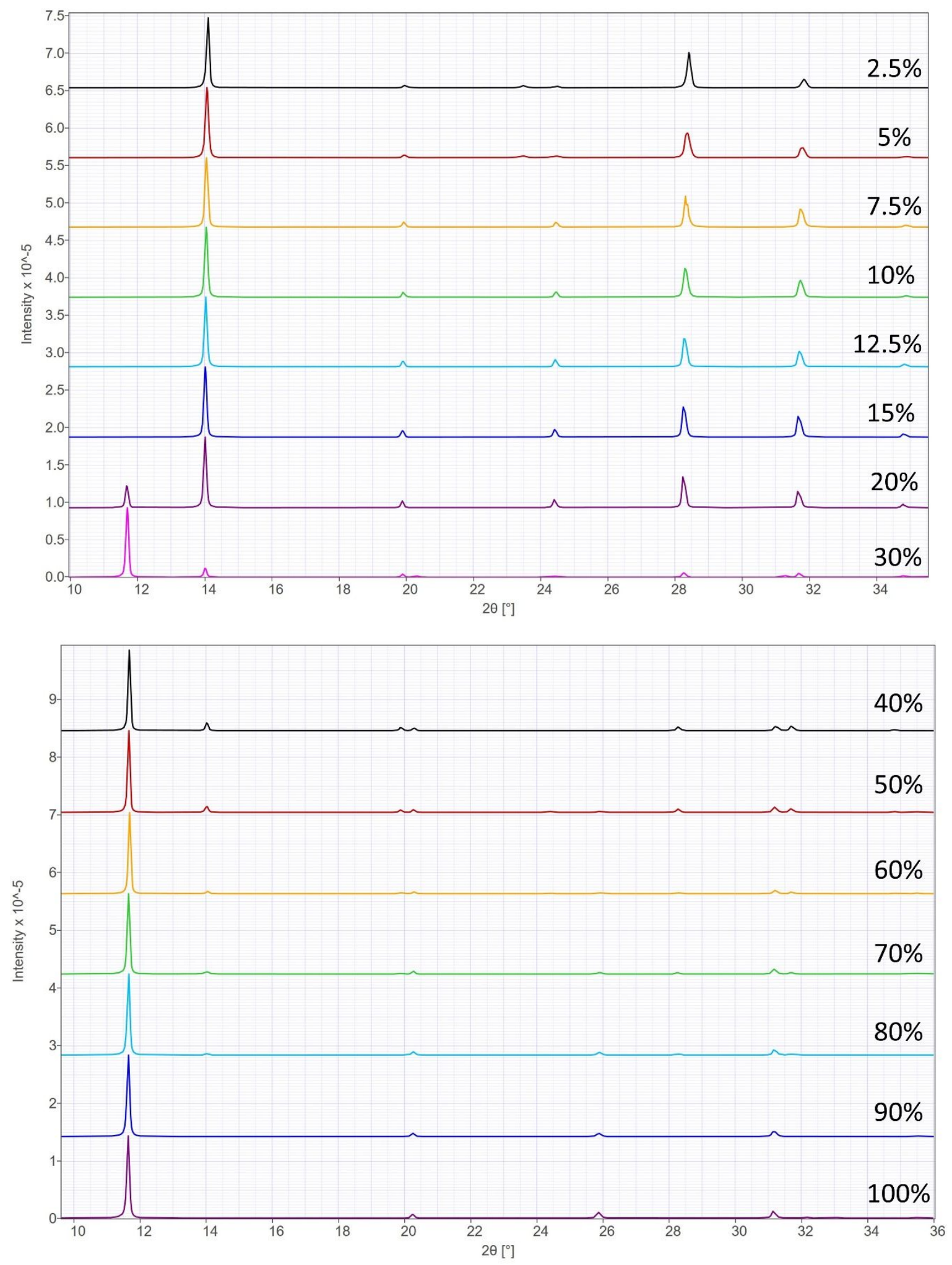

Figure S6: PXRD patterns of varying substitution percentages of DMA (dimethylammonium) for MA (methylammonium) in $\mathrm{MA}_{1-\mathrm{x}} \mathrm{DMA}_{\mathrm{x}} \mathrm{Pbl}_{3}$ materials. 

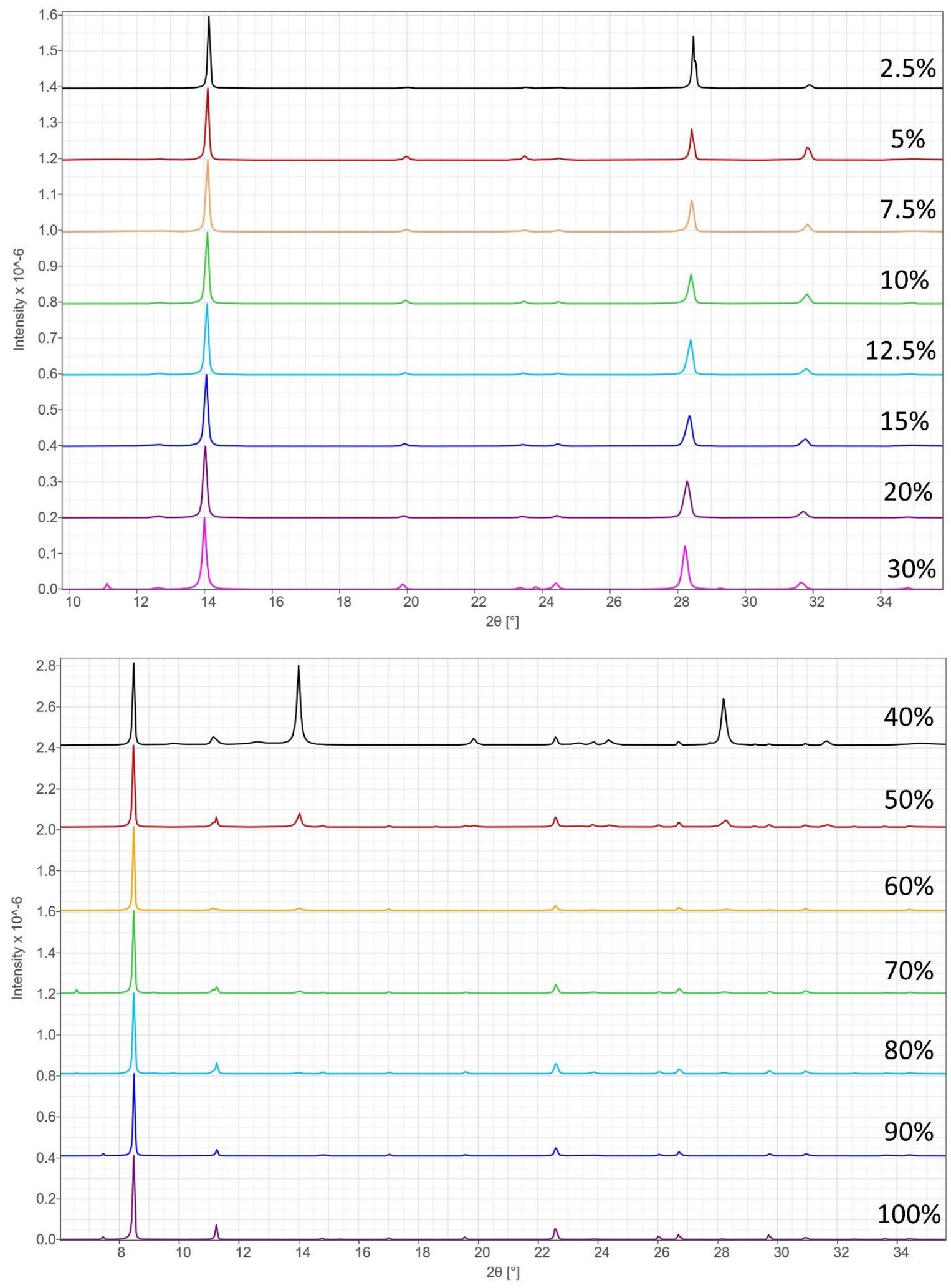

Figure S7: PXRD patterns of varying substitution percentages of GA (guanidinium) for MA (methylammonium) in $\mathrm{MA}_{1-\mathrm{x}} \mathrm{GA}_{\mathrm{x}} \mathrm{Pbl}_{3}$ materials 
a.
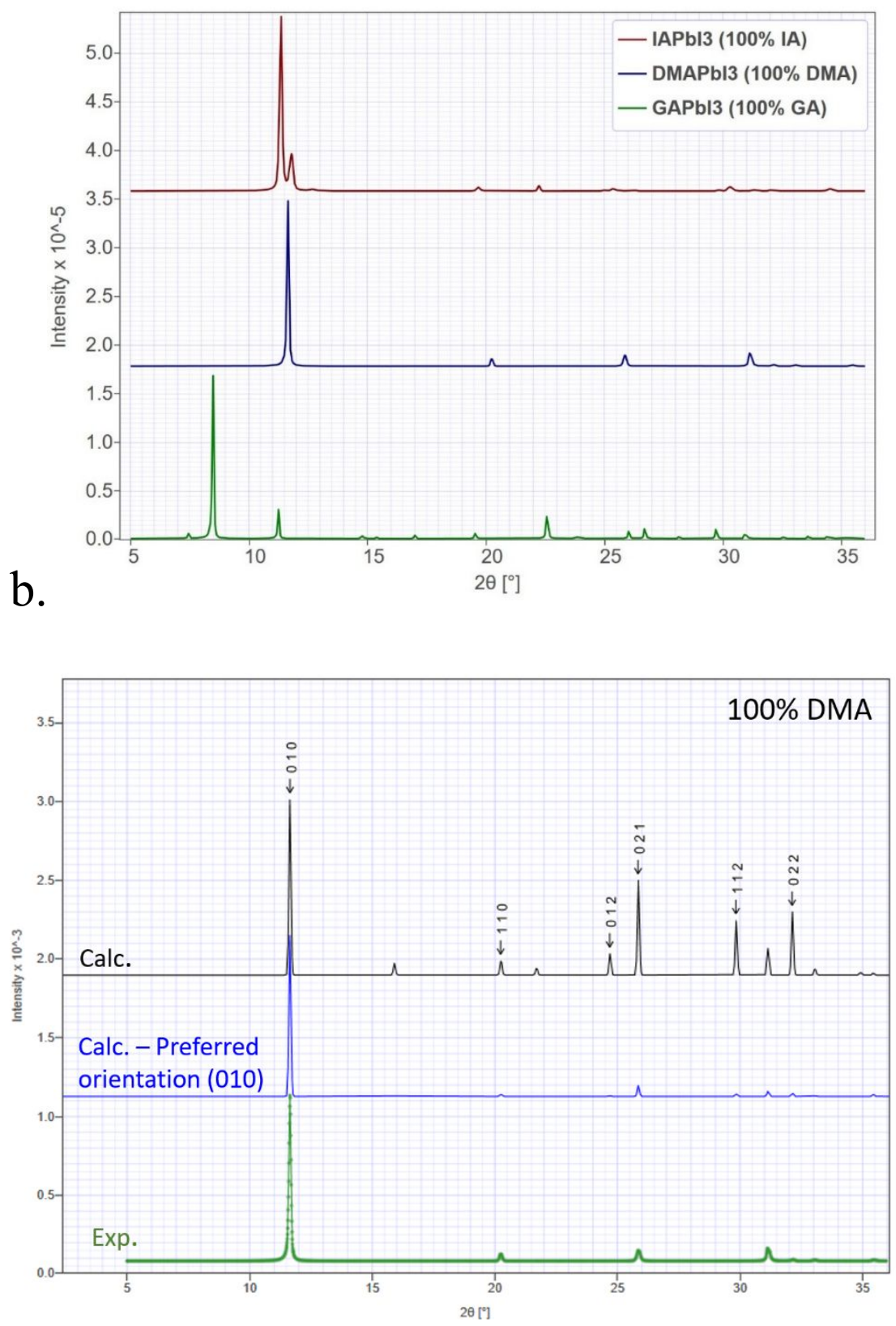

Figure S8: a. PXRD patterns of $100 \%$ large cation substituted compounds (0\% MA). b. Experimental PXRD pattern of $100 \%$ DMA (bottom, green) with calculated pattern ${ }^{1}$ (top, black) and similuated pattern with prefererd oritnetation along the (010) plane (middle, blue).

${ }^{1}$ Mancini, A.; Quadrelli, P.; Amoroso, G.; Milanese, C.; Boiocchi, M.; Sironi, A.; Patrini, M.; Guizzetti, G.; Malavasi, L., Synthesis, structural and optical characterization of $\mathrm{APbX}_{3}(\mathrm{~A}=$ methylammonium, dimethylammonium, trimethylammonium; $\mathrm{X}=\mathrm{I}, \mathrm{Br}, \mathrm{Cl})$ hybrid organic-inorganic materials. Journal of Solid State Chemistry 2016, 240 (1), 55-60. 

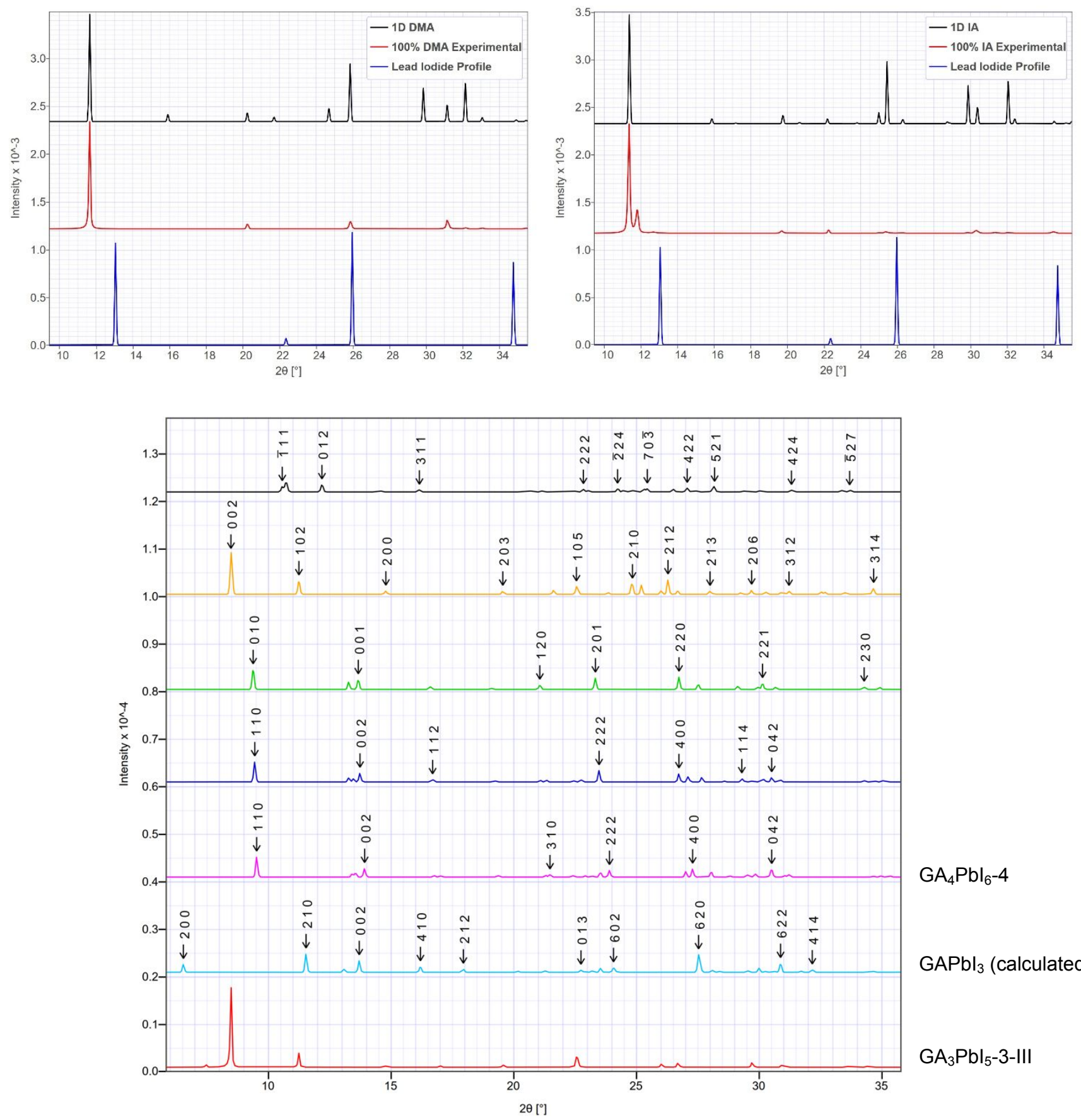

$\mathrm{GA}_{4} \mathrm{Pbl}_{6}-4$

$\mathrm{GAPbl}_{3}$ (calculated)

$\mathrm{GA}_{3} \mathrm{Pbl}_{5}-3-\mathrm{III}$

Figure S9: PXRD patterns of films made with $100 \%$ of each large cation (DMA, IA, and GA) al $\mathrm{GA}_{3} \mathrm{Pbl} \mathrm{I}_{5}-3-\mathrm{II}$ and iodide compared to common crystal structure of this mixture of ions reported in literature. Although all three films were a similar yellow color to the lead iodide precursor (blue), none appear to have anv lead iodide. DMA and IA have similar structures to the one-dimensional mixtures reported $\mathrm{GA}_{3} \mathrm{Pbl}_{5}-3$ although with fewer peaks, which may be a result of preferential orientation. Our experimental spectra for $100 \%$ GA closely matches the calculated $\mathrm{GAPbl}_{3}$ pattern.

$\mathrm{GA}_{2} \mathrm{Pbl}_{4}-\mathrm{II}(@ 310 \mathrm{~K})$ 
a.

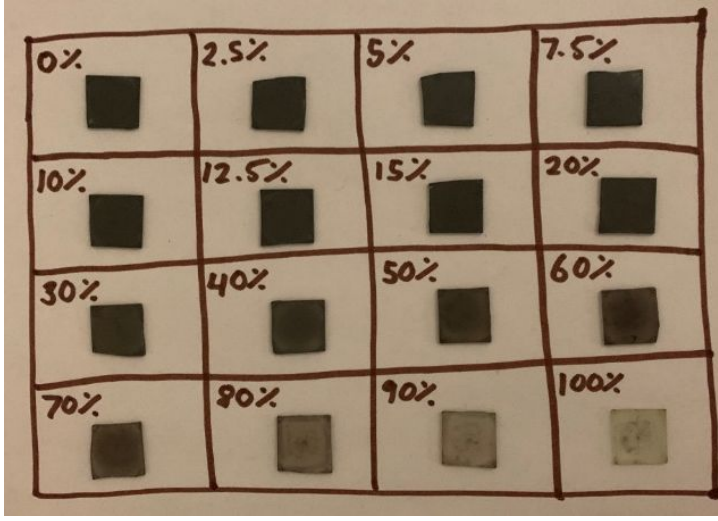

c.
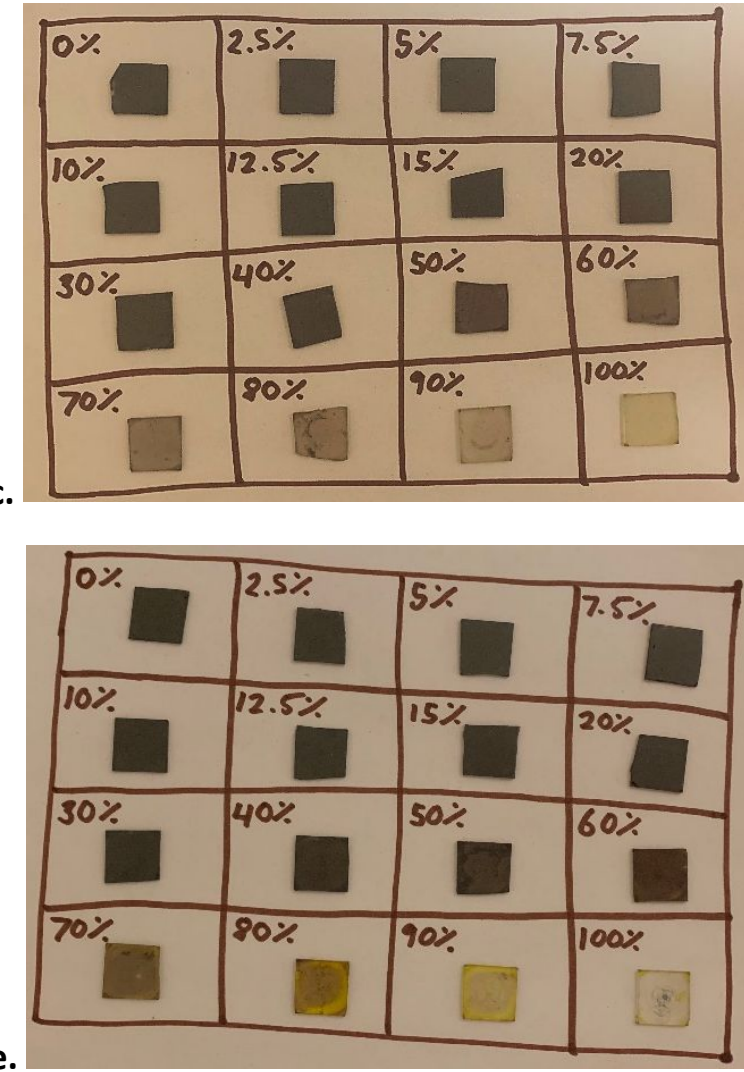

b.

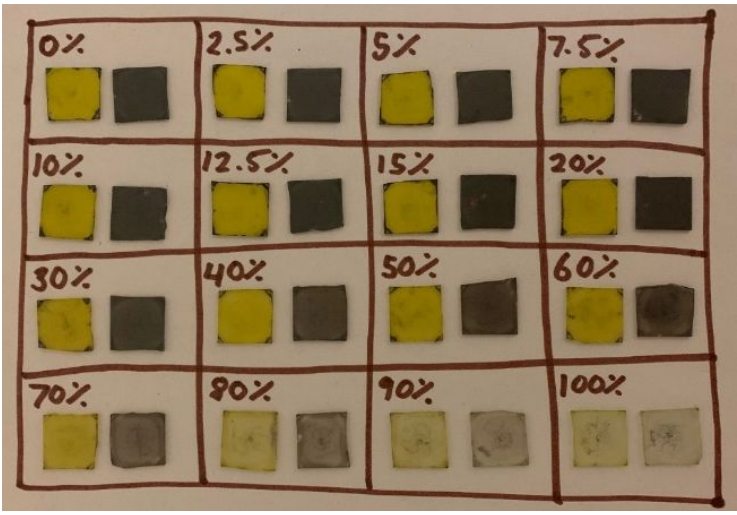

d.
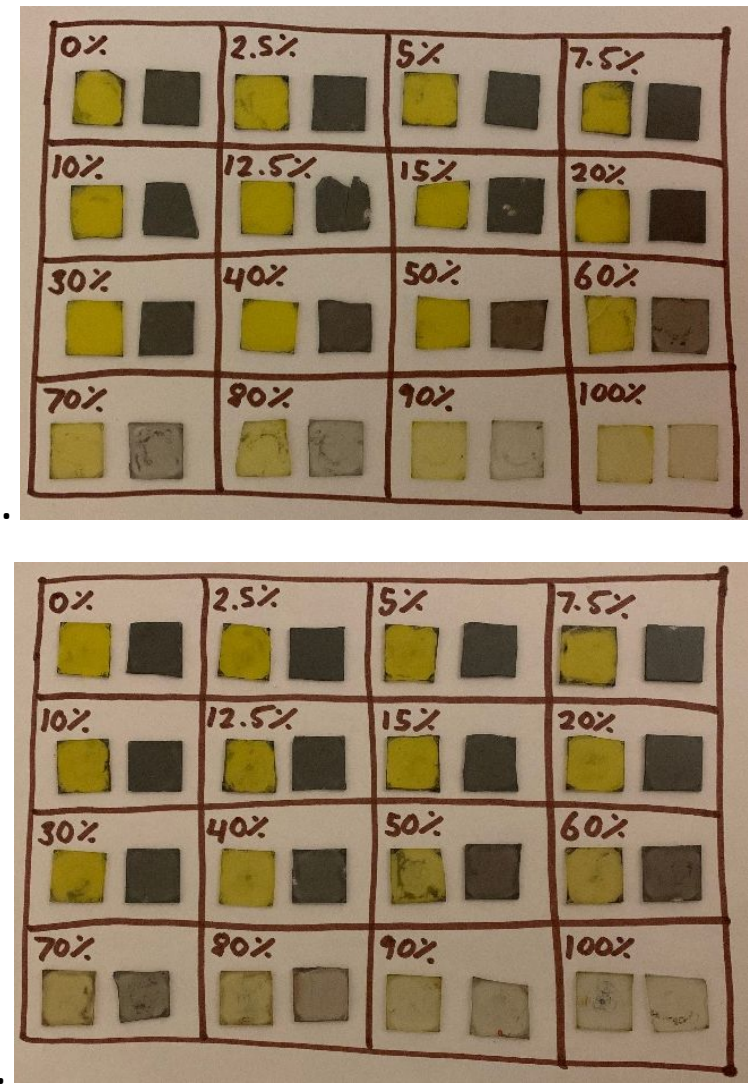

Figure S10: Increasing \% large cation (LA) substituted samples $0 \%=\mathrm{MAPbl}_{3}, 100 \%=\mathrm{LAPbl}_{3}$. Thin films on PEDOT:PSS-coated glass substrates. $a$. and $b .=$ DMA, $c$. and d. = IA, e. and f. = GA. Left $(a, c, e)$ : Freshly made samples. Right $(b, d, f)$ : Samples after $24 \mathrm{~h}$. Samples on left in each cell have been exposed to light for $24 \mathrm{~h}$, samples on right are controls that have been kept in the dark in same ambient conditions (ambient air, temperature, humidity). Dark controls showed no discernable degradation over the $24 \mathrm{~h}$ period by PXRD analysis. 


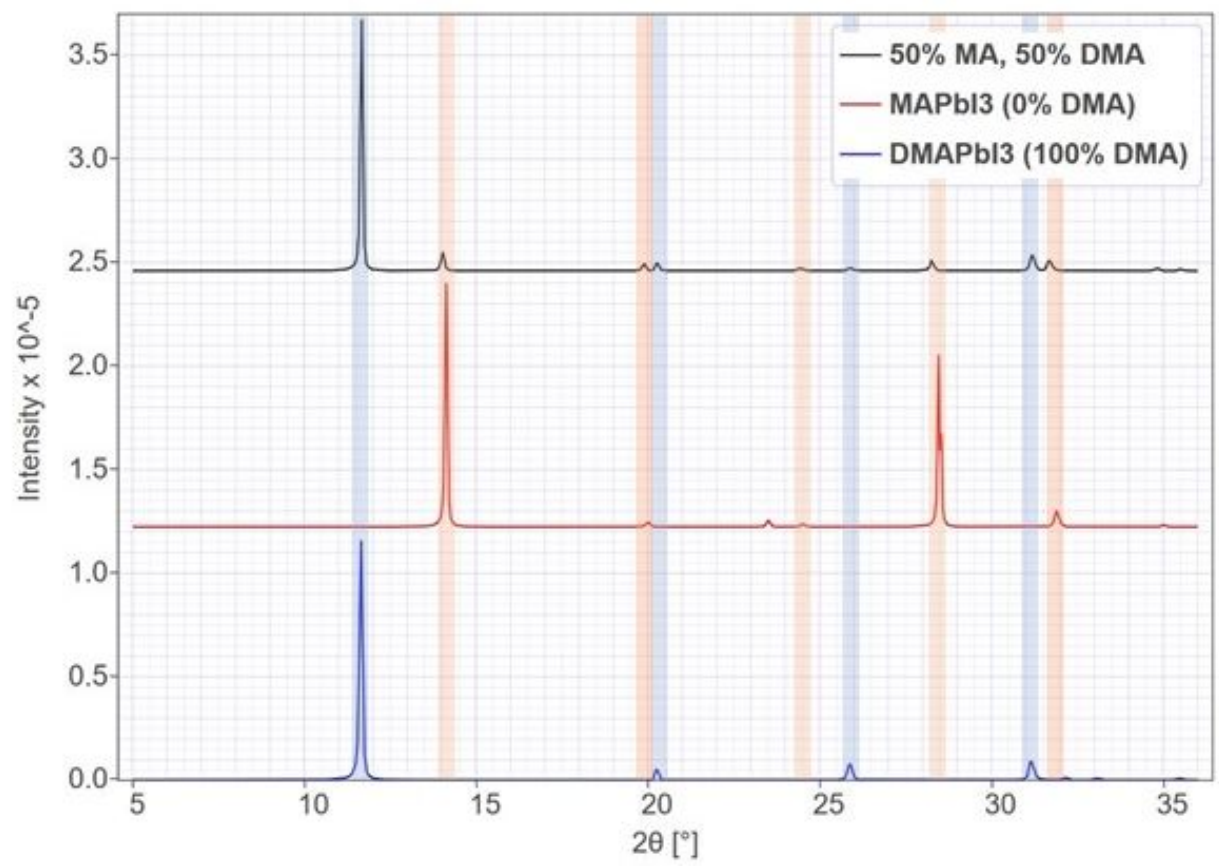

Figure S11: PXRD patterns of $\mathrm{MA}_{0.5} \mathrm{DMA}_{0.5} \mathrm{Pbl}_{3}$ (50\% DMA substitution), $\mathrm{MAPbl}_{3}$ (0\% DMA substitution), and $\mathrm{DMAPbl}_{3}$ (100\% DMA substitution). Both the $0 \%$ and $100 \%$ phases are present together in $50 \%$ sample. This is similar for IA. GA has some unassigned peaks in mid substitution percentages. 

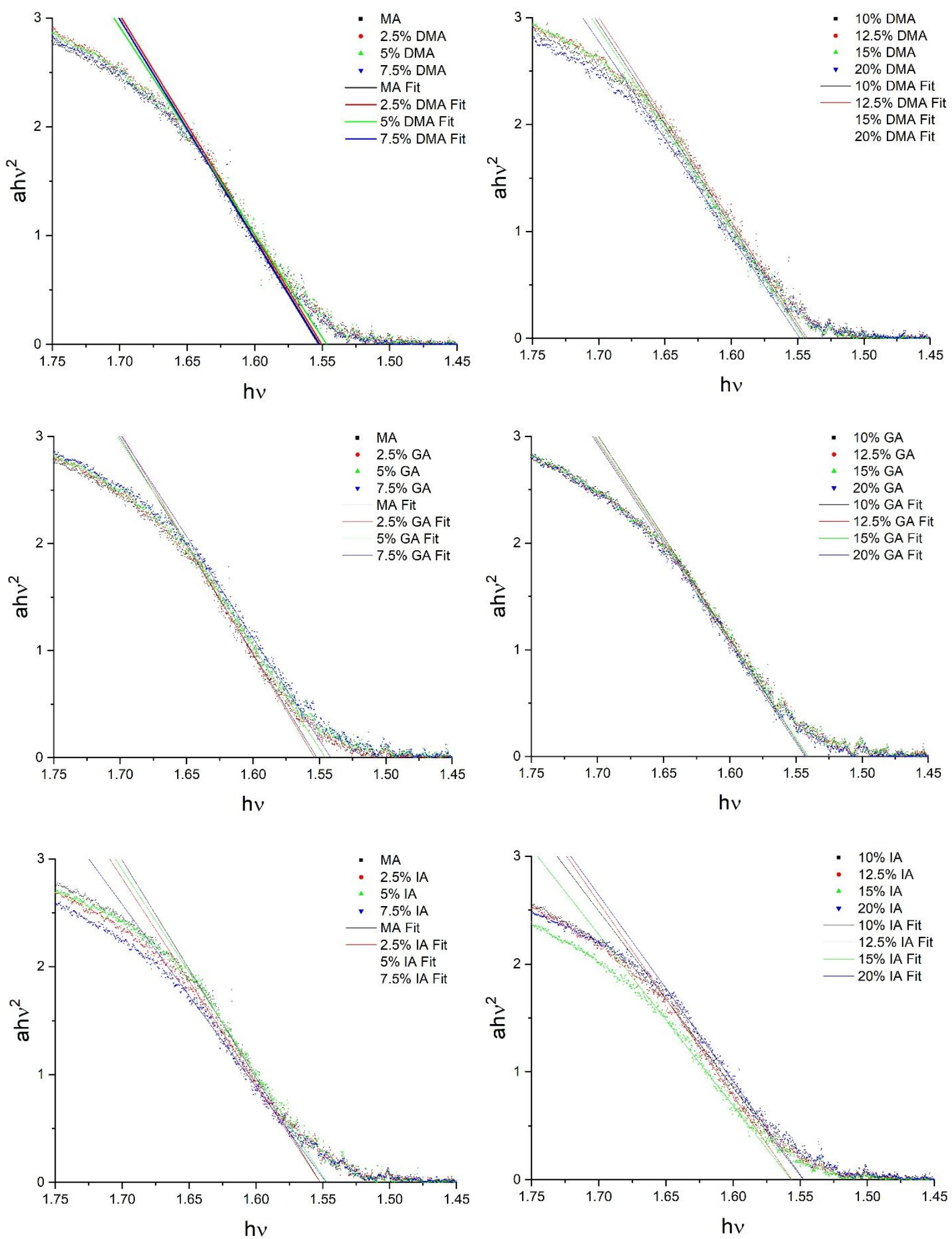

Figure S12: Tauc plots from UV-Vis absorption data of 0-20\% substituted films used to calculate band gap. 

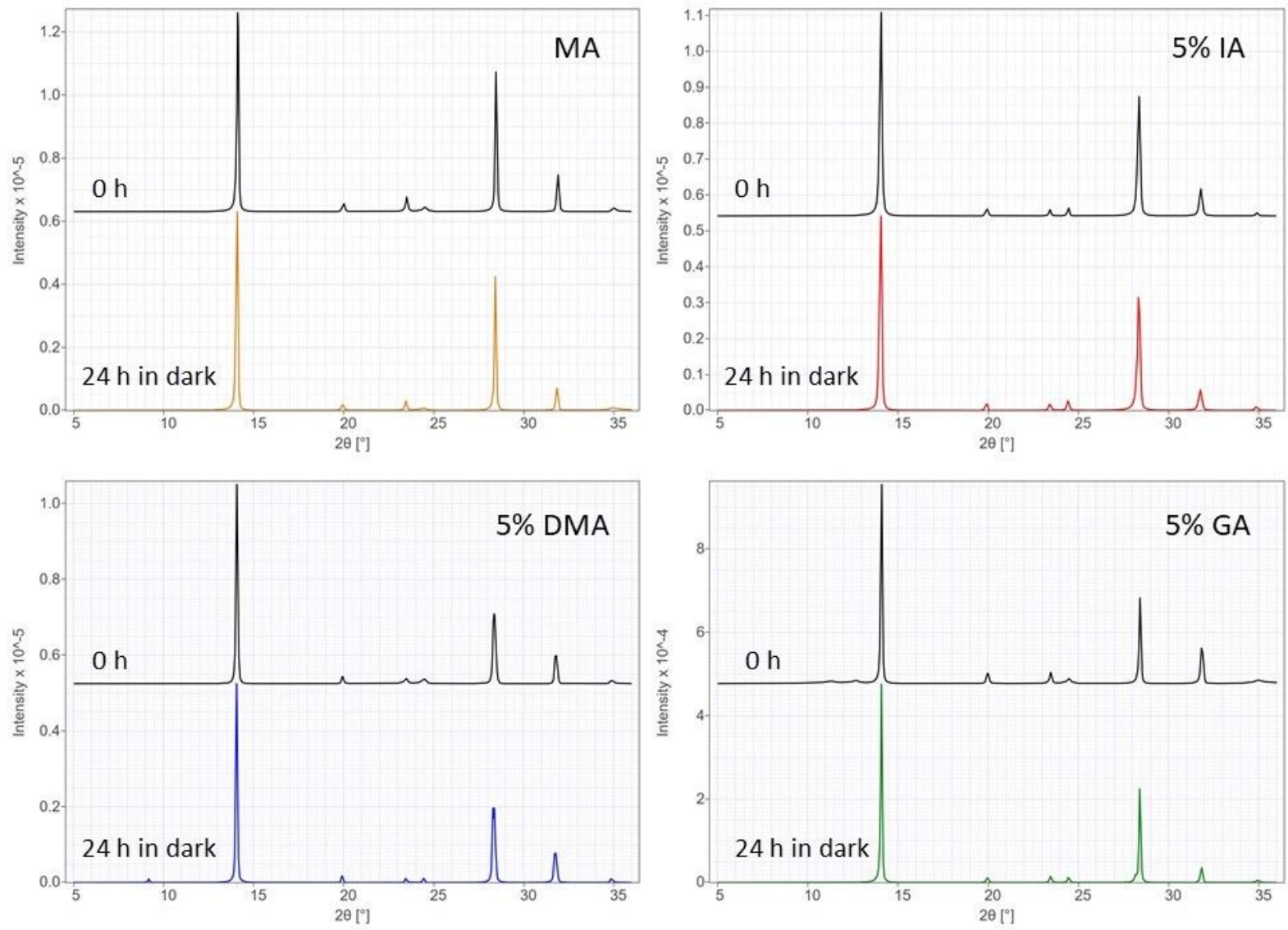

Figure S13: $\mathrm{MAPbl}_{3}$ and $5 \%$ substitution control samples at $0 \mathrm{~h}$ and after $24 \mathrm{~h}$ in dark. 

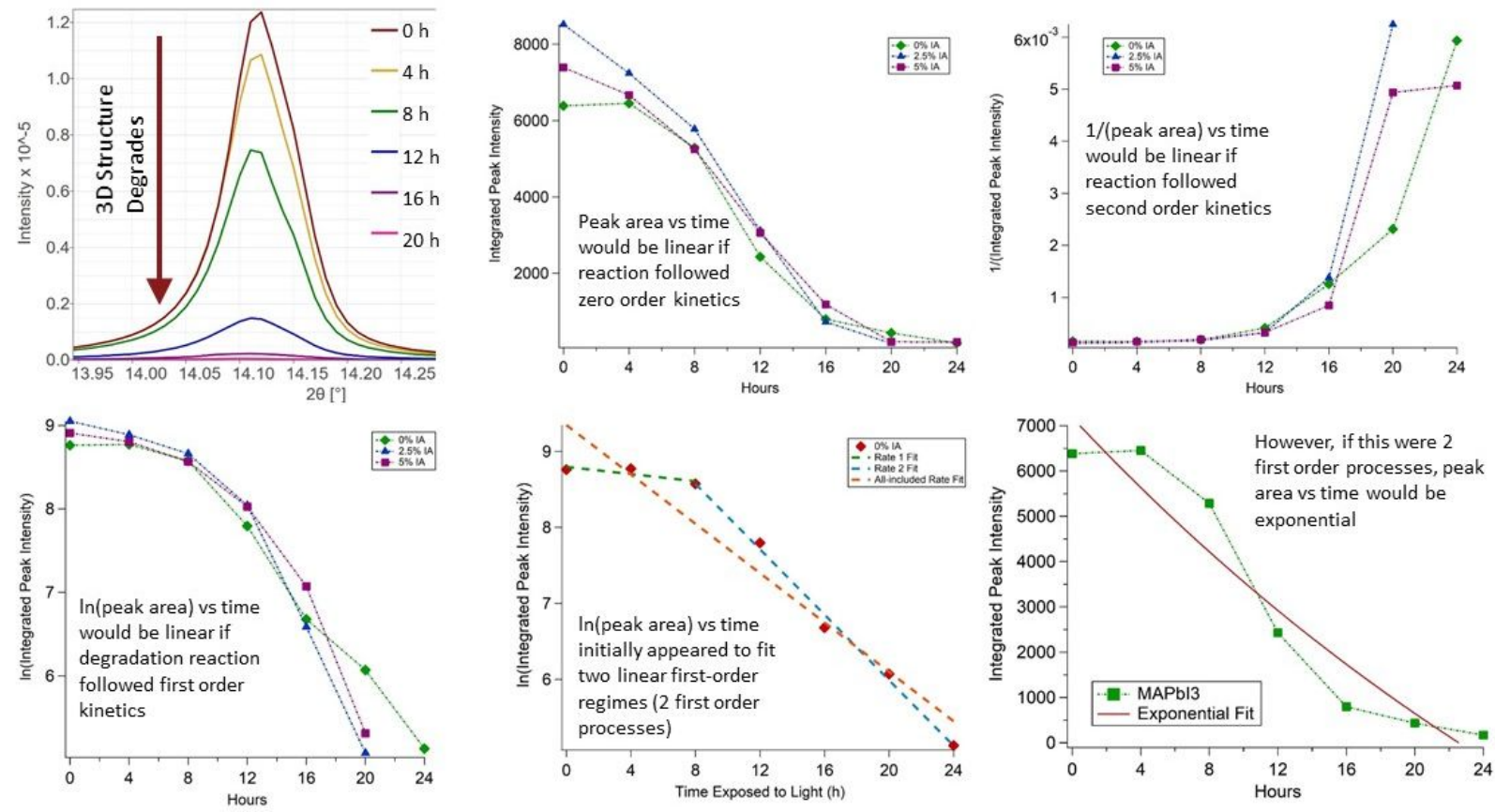

Figure S14: Simple zero, first, and second-order kinetic models were ruled out before applying more complex models. 
Table S1: Lattice parameters of 0-20\% large cation substituted compounds

\begin{tabular}{|c|c|c|c|c|c|c|c|c|}
\hline & MA & $\begin{array}{l}2.5 \% \\
\text { DMA }\end{array}$ & $\begin{array}{l}5 \% \\
\text { DMA }\end{array}$ & $\begin{array}{l}7.5 \% \\
\text { DMA }\end{array}$ & $\begin{array}{l}10 \% \\
\text { DMA }\end{array}$ & $\begin{array}{l}12.5 \% \\
\text { DMA }\end{array}$ & $\begin{array}{l}15 \% \\
\text { DMA }\end{array}$ & $\begin{array}{l}20 \% \\
\text { DMA }\end{array}$ \\
\hline $\mathrm{a}, \AA$ & 8.8670 & 8.9105 & 8.9110 & 8.9097 & 8.9114 & 8.9137 & 8.9202 & 8.9216 \\
\hline b, Å & 8.8670 & 8.9105 & 8.9110 & 8.9097 & 8.9114 & 8.9137 & 8.9202 & 8.9216 \\
\hline c, $\AA$ & 12.5314 & 12.5533 & 12.5967 & 12.5967 & 12.6019 & 12.6116 & 12.6194 & 12.6231 \\
\hline$\alpha,{ }^{\circ}$ & 90 & 90 & 90 & 90 & 90 & 90 & 90 & 90 \\
\hline$\beta,{ }^{\circ}$ & 90 & 90 & 90 & 90 & 90 & 90 & 90 & 90 \\
\hline$v_{i^{\circ}}$ & 90 & 90 & 90 & 90 & 90 & 90 & 90 & 90 \\
\hline Volume & 985.27 & 996.69 & 1000.25 & 999.96 & 1000.77 & 1002.04 & 1004.13 & 1004.74 \\
\hline
\end{tabular}

\begin{tabular}{|l|l|l|l|l|l|l|l|l|}
\hline & MA & 2.5\% GA & 5\% GA & 7.5\% GA & 10\% GA & 12.5\% GA & 15\% GA & 20\% GA \\
\hline a, $\AA$ & 8.8670 & 8.8784 & 8.8770 & 8.8973 & 8.8923 & 8.8992 & 8.9085 & 8.9194 \\
\hline $\mathbf{b , ~} \AA$ & 8.8670 & 8.8784 & 8.8770 & 8.8973 & 8.8923 & 8.8992 & 8.9085 & 8.9194 \\
\hline $\mathbf{c}, \AA$ & 12.5314 & 12.5405 & 12.5437 & 12.5617 & 12.5636 & 12.5729 & 12.5932 & 12.6145 \\
\hline $\boldsymbol{\alpha}^{\circ}$ & 90 & 90 & 90 & 90 & 90 & 90 & 90 & 90 \\
\hline $\boldsymbol{\beta}^{\circ}$ & 90 & 90 & 90 & 90 & 90 & 90 & 90 & 90 \\
\hline $\boldsymbol{\gamma}^{\circ}$ & 90 & 90 & 90 & 90 & 90 & 90 & 90 & 90 \\
\hline Volume & 985.27 & 988.51 & 988.44 & 994.41 & 993.44 & 995.71 & 999.41 & 1003.55 \\
\hline
\end{tabular}

\begin{tabular}{|l|l|l|l|l|l|l|l|l|}
\hline & MA & 2.5\% IA & 5\% IA & 7.5\% IA & 10\% IA & 12.5\% IA & 15\% IA & 20\% IA \\
\hline a, $\AA$ & 8.8670 & 8.8890 & 8.8961 & 8.9069 & 8.9142 & 8.9135 & 8.9058 & 8.9145 \\
\hline $\mathbf{b , ~} \AA$ & 8.8670 & 8.8890 & 8.8961 & 8.9069 & 8.9142 & 8.9135 & 8.9058 & 8.9145 \\
\hline $\mathbf{c}, \AA$ & 12.5314 & 12.5633 & 12.5803 & 12.5945 & 12.6096 & 12.6060 & 12.5934 & 12.6071 \\
\hline $\boldsymbol{\alpha}^{\circ}$ & 90 & 90 & 90 & 90 & 90 & 90 & 90 & 90 \\
\hline $\boldsymbol{\beta}^{\circ}$ & 90 & 90 & 90 & 90 & 90 & 90 & 90 & 90 \\
\hline $\mathbf{r}^{\circ}$ & 90 & 90 & 90 & 90 & 90 & 90 & 90 & 90 \\
\hline Volume $^{\circ}$ & 985.27 & 992.69 & 995.60 & 999.16 & 1002.00 & 1001.56 & 998.82 & 1001.87 \\
\hline
\end{tabular}


Table S2: Band gaps of substituted compounds calculated from tauc plots of UV-Vis spectrophotometry data.

\begin{tabular}{|l|l|l|l|}
\hline \% Large Cation & IA Band Gap (eV) & GA Band Gap (eV) & DMA Band Gap (eV) \\
\hline $0.0 \%$ & 1.554 & 1.554 & 1.554 \\
\hline $2.5 \%$ & 1.552 & 1.554 & 1.549 \\
\hline $5.0 \%$ & 1.546 & 1.545 & 1.544 \\
\hline $7.5 \%$ & 1.549 & 1.542 & 1.552 \\
\hline $10.0 \%$ & 1.545 & 1.540 & 1.544 \\
\hline $12.5 \%$ & 1.557 & 1.541 & 1.546 \\
\hline $15.0 \%$ & 1.559 & 1.552 & 1.552 \\
\hline $20.0 \%$ & 1.548 & 1.543 & 1.548 \\
\hline
\end{tabular}

Table S3: Rate coefficients $k$ of light-induced degradation reaction and percent decrease in $k$ for each substituted sample compared to its $\mathrm{MAPbl}_{3}$ control ( $0 \%$ substitution in top row).

\begin{tabular}{|l|l|l|l|l|l|l|}
\hline & \multicolumn{2}{|l|}{ IA } & \multicolumn{2}{l|}{ DMA } & GA \\
\hline $\begin{array}{l}\text { \% Cation } \\
\text { Substitution }\end{array}$ & $\boldsymbol{k}\left(\mathbf{h}^{-\mathbf{1}}\right)$ & $\begin{array}{l}\text { \% decrease in } \boldsymbol{k} \\
\text { from } \mathbf{M A P b I}_{\mathbf{3}}\end{array}$ & $\boldsymbol{k}\left(\mathbf{h}^{-\mathbf{1}}\right)$ & $\begin{array}{l}\text { \% decrease in } \boldsymbol{k} \\
\text { from } \mathbf{M A P b I}_{\mathbf{3}}\end{array}$ & $\boldsymbol{k}\left(\mathbf{h}^{-1}\right)$ & $\begin{array}{l}\text { \% decrease in } \\
\boldsymbol{k} \text { from } \mathbf{M A P b I}_{\mathbf{3}}\end{array}$ \\
\hline $\mathbf{0 . 0 \%}$ & 0.43463 & ---- & 0.55122 & ---- & 0.63056 & ---- \\
\hline $\mathbf{2 . 5 \%}$ & 0.34249 & $21.20 \%$ & 0.47096 & $14.56 \%$ & 0.50945 & $19.21 \%$ \\
\hline $\mathbf{5 . 0 \%}$ & 0.31384 & $27.79 \%$ & 0.42335 & $23.20 \%$ & 0.23656 & $62.48 \%$ \\
\hline $\mathbf{7 . 5 \%}$ & 0.21184 & $51.26 \%$ & 0.39979 & $27.47 \%$ & 0.27871 & $55.80 \%$ \\
\hline $\mathbf{1 0 . 0 \%}$ & 0.30499 & $29.83 \%$ & 0.44917 & $18.51 \%$ & 0.24689 & $60.85 \%$ \\
\hline $\mathbf{1 2 . 5 \%}$ & 0.31102 & $28.44 \%$ & 0.42251 & $23.35 \%$ & 0.31028 & $50.79 \%$ \\
\hline $\mathbf{1 5 . 0 \%}$ & 0.29119 & $33.00 \%$ & 0.37239 & $32.44 \%$ & 0.30805 & $51.15 \%$ \\
\hline $\mathbf{2 0 . 0 \%}$ & 0.28757 & $33.84 \%$ & 0.34747 & $36.96 \%$ & 0.37002 & $41.32 \%$ \\
\hline
\end{tabular}


Table S4: Area of most intense PXRD from 1D degradation product $(I A=(110), D M A=(010), G A=(120))$ as a function of time.

\begin{tabular}{|l|l|l|l|l|l|l|l|}
\hline $\mathbf{I A}$ & $\mathbf{0} \mathbf{h}$ & $\mathbf{4} \mathbf{h}$ & $\mathbf{8} \mathbf{h}$ & $\mathbf{1 2} \mathbf{h}$ & $\mathbf{1 6} \mathbf{h}$ & $\mathbf{2 0} \mathbf{h}$ & $\mathbf{2 4} \mathbf{h}$ \\
\hline $\mathbf{0 . 0 \%}$ & 0 & 0 & 0 & 0 & 0 & 0 & 0 \\
\hline $\mathbf{2 . 5 \%}$ & 0 & 0 & 0 & 0 & 0 & 0 & 0 \\
\hline $\mathbf{5 . 0 \%}$ & 0 & 0 & 0 & 0 & 0 & 0 & 0 \\
\hline $\mathbf{7 . 5 \%}$ & 0 & 0 & 0 & 0 & 0 & 0 & 0 \\
\hline $\mathbf{1 0 . 0 \%}$ & 0 & 0 & 0 & 0 & 0 & 0 & 0 \\
\hline $\mathbf{1 2 . 5 \%}$ & 563.62 & 310.78 & 315.4 & 264.7 & 1430.44 & 293.74 & 508.06 \\
\hline $\mathbf{1 5 . 0 \%}$ & 452.08 & 326.5 & 407.97 & 516.12 & 217.89 & 784.75 & 626.91 \\
\hline $\mathbf{2 0 . 0 \%}$ & 1267.89 & 1164.47 & 1187.72 & 1159.69 & 682.43 & 482.13 & 801.65 \\
\hline
\end{tabular}

\begin{tabular}{|l|l|l|l|l|l|l|}
\hline DMA & $\mathbf{O} \mathbf{h}$ & $\mathbf{4} \mathbf{h}$ & $\mathbf{8} \mathbf{h}$ & $\mathbf{1 2} \mathbf{h}$ & $\mathbf{1 6} \mathbf{h}$ & $\mathbf{2 0} \mathbf{h}$ \\
\hline $\mathbf{0 . 0 \%}$ & 0 & 0 & 0 & 0 & 0 & 0 \\
\hline $\mathbf{2 . 5 \%}$ & 0 & 0 & 0 & 0 & 0 & 0 \\
\hline $\mathbf{5 . 0 \%}$ & 0 & 0 & 0 & 0 & 0 & 0 \\
\hline $\mathbf{7 . 5 \%}$ & 0 & 0 & 0 & 0 & 0 & 0 \\
\hline $\mathbf{1 0 . 0 \%}$ & 0 & 0 & 0 & 0 & 0 & 0 \\
\hline $\mathbf{1 2 . 5 \%}$ & 0 & 0 & 0 & 0 & 0 & 0 \\
\hline $\mathbf{1 5 . 0 \%}$ & 0 & 0 & 0 & 145.76 & 108.52 & 0 \\
\hline $\mathbf{2 0 . 0 \%}$ & 808.48 & 700.51 & 599.56 & 621.03 & 468.75 & 231.61 \\
\hline
\end{tabular}

\begin{tabular}{|l|l|l|l|l|l|l|}
\hline $\mathbf{G A}$ & $\mathbf{0} \mathbf{h}$ & $\mathbf{4} \mathbf{h}$ & $\mathbf{8} \mathbf{h}$ & $\mathbf{1 2} \mathbf{h}$ & $\mathbf{1 6} \mathbf{h}$ & $\mathbf{2 0} \mathbf{h}$ \\
\hline $\mathbf{0 . 0} \%$ & 0 & 0 & 0 & 0 & 0 & 0 \\
\hline $\mathbf{2 . 5 \%}$ & 0 & 0 & 0 & 0 & 0 & 0 \\
\hline $\mathbf{5 . 0 \%}$ & 0 & 0 & 0 & 0 & 0 & 0 \\
\hline $\mathbf{7 . 5 \%}$ & 0 & 0 & 0 & 90.99 & 133.65 & 252.76 \\
\hline $\mathbf{1 0 . 0 \%}$ & 0 & 0 & 90.97 & 223.01 & 283.31 & 317.28 \\
\hline $\mathbf{1 2 . 5 \%}$ & 0 & 0 & 107.28 & 247.36 & 317.46 & 502.49 \\
\hline $\mathbf{1 5 . 0 \%}$ & 0 & 0 & 93.34 & 304.09 & 424.11 & 484.81 \\
\hline $\mathbf{2 0 . 0 \%}$ & 0 & 158.14 & 464.24 & 818.91 & 917.18 & 945.09 \\
\hline
\end{tabular}

